Article

\title{
Bartonella spp. Prevalence (Serology, Culture, and PCR) in Sanitary Workers in La Rioja Spain
}

\author{
Aránzazu Portillo ${ }^{1}$, Ricardo Maggi ${ }^{2,3}$, José A. Oteo ${ }^{1}$, Julie Bradley ${ }^{3}$, Lara García-Álvarez ${ }^{1}$, \\ Montserrat San-Martín ${ }^{4}$ (D), Xavier Roura ${ }^{5}$ (D) and Edward Breitschwerdt ${ }^{2,3, *(D)}$ \\ 1 Departamento de Enfermedades Infecciosas, Hospital U. San Pedro-Centro de Investigación Biomédica de \\ La Rioja (CIBIR), 26006 Logroño, La Rioja, Spain; aportillo@riojasalud.es (A.P.); jaoteo@riojasalud.es (J.A.O.); \\ lgalvarez.ext@riojasalud.es (L.G.-Á.) \\ 2 Galaxy Diagnostics, Research Triangle Park, Morrisville, NC 27709, USA; rgmaggi@ncsu.edu \\ 3 Intracellular Pathogens Research Laboratory, Comparative Medicine Institute, College of Veterinary \\ Medicine, North Carolina State University, Raleigh, NC 27695, USA; Julie_Bradley@ncsu.edu \\ 4 Faculty of Social Sciences, University of Granada, 52006 Melilla, Spain; momartin@ugr.es \\ 5 Hospital Clínic Veterinari, Universitat Autònoma de Barcelona, 08193 Bellaterra, Barcelona, Spain; \\ xavier.roura@uab.cat \\ * Correspondence: ebbreits@ncsu.edu; Tel.: +1-919-513-8277
}

Received: 17 February 2020; Accepted: 2 March 2020; Published: 4 March 2020

\begin{abstract}
Bartonella spp. are increasingly implicated in association with a spectrum of zoonotic infectious diseases. One hundred sanitary workers in La Rioja, Spain, completed a questionnaire and provided blood specimens for Bartonella spp. serology and Bartonella Alpha-Proteobacteria growth medium (BAPGM) enrichment blood culture/PCR. Six immunofluorescence assays (IFA) were performed and aseptically obtained blood specimens were inoculated into liquid BAPGM and subcultured onto blood agar plates. Bartonella DNA was amplified using conventional and real-time PCR assays. The Bartonella spp., strain, or genotype was determined by DNA sequencing. Bartonella seroreactivity was documented in $83.1 \%$ and bloodstream infection in $21.6 \%$ of participants. Bartonella henselae, B. vinsonii subsp. berkhoffii genotypes I and III, and B. quintana were identified. IFA seroreactivity and PCR positivity were not statistically associated with self-reported symptoms. Our results suggest that exposure to and non-clinical infection with Bartonella spp. may occur more often than previously suspected in the La Rioja region.
\end{abstract}

Keywords: Bartonella Alpha-Proteobacteria Growth Medium (BAPGM), B. henselae; B. quintana; B. vinsonii subsp. berkhoffii; $B$. koehlerae; sanitary workers

\section{Introduction}

The genus Bartonella comprises fastidious Gram-negative, slow growing and facultative intracellular bacteria belonging to the Alpha-2 subgroup of the class Proteobacteria, Order Rhizobiales. These microorganisms are most often transmitted to humans through animal bites or scratches (cats, dogs and other animals), or by scratch inoculation of infected flea and body louse feces [1]. In addition, sand-fly vector-competence was experimentally proven for transmission of Bartonella bacilliformis in Peru during the last century [2]. Other arthropods such as ticks, head lice, bedbugs, bat flies and mites have been associated with human Bartonella spp. infections, but their role as competent vectors requires further confirmation [3-7]. Bartonella spp. can survive within different hosts and reservoirs (arthropods, mammals, humans) for months to years. This genus has been increasingly associated with a wide spectrum of zoonotic emerging and reemerging infectious diseases. The number of validated species (more than 35), potential species (at least 17) and subspecies (at least three) continues to increase $<\mathrm{http}: / / w w w \cdot b a c t e r i o \cdot n e t / b a r t o n e l l a . h t m l>$. Some 
species, such as B. bacilliformis, cause potentially life threatening illness, but are limited geographically by the transmitting vector, whereas other species such as B. quintana are transmitted under poor hygienic conditions throughout the world. Flea-transmitted $B$. henselae is the most frequent etiological agent of sub-acute and chronic lymphadenopathy named cat-scratch disease (CSD) in children and teenagers and occurs throughout the world. A subset of CSD patients develop severe or systemic disease manifestations, including endocarditis, osteomyelitis, granulomatous hepatitis and hepatosplenic abscess [8-12]. Apart from these 'classical' species, at least 15 Bartonella spp. have been associated with human diseases (e.g., Bartonella vinsonii subsp. berkhoffii or B. vinsonii subsp. arupensis) or, at least, have been detected in humans (e.g. B. vinsonii subsp. vinsonii).

Seemingly, all Bartonella spp. may represent potential opportunistic pathogens for infections in animals and human patients $[13,14]$. Under a One Health prism, the collective understanding of Bartonella epidemiology and pathogenesis continues to change [13]. After recent studies in Brazil documented Bartonella infection (positive PCR/DNA sequencing results) in healthy blood donors [15-17], the authors initiated this study to assess the prevalence of Bartonella spp. infection in sanitary workers in a localized region of Spain. Previously, a high prevalence of Bartonella spp. antibodies, as well as DNA of these bacteria, were reported in blood samples collected from veterinary workers in Spain [18]. Compared to veterinary workers, who are frequently exposed to arthropod vectors and infected animals, sanitary workers in La Rioja were not considered to be at a high risk for exposure to or infection with Bartonella spp. In addition to microbiological testing, the potential relationship of Bartonella spp. antibodies or blood stream infection (as assessed by DNA amplification and sequencing) with minor or nonspecific, self-reported symptoms, such as fatigue or insomnia was examined.

\section{Results}

\subsection{Subject Recruitment}

Study participants were recruited from among physicians, nurses, researchers, medical students, and administrative personnel who worked at the Center of Biomedical Research (CIBIR) or San Pedro's University Hospital at La Rioja (Northern Spain). The buildings housing these facilities are adjacent to each other and personnel share a common café. Physicians, medical students and nurses have contact with patients diagnosed with CSD or other Bartonella infections. The CIBIR houses the Center of Rickettsiosis and Arthropod-borne Diseases, where researchers support the diagnosis of arthropod (especially tick borne, but also flea, mite, and mosquitoes) associated diseases. Clinical specimens are tested using serological and molecular assays for Bartonella, Borrelia, Coxiella, Rickettsia spp. and other pathogens. Bartonella spp. are not routinely cultured and are infrequently isolated. Isolates of $B$. quintana and B. vinsonii subsp. berkhoffii genotypes have not been cultured in the research laboratories.

After excluding three individuals who had recently taken antimicrobials, 97 sanitary workers were included in the study. A total of 194 participant samples (97 EDTA-blood and 97 sera) and the accompanying questionnaires were used to generate data reported in the results. Questionnaire demographic and risk exposure characteristics are provided in Table 1. Trekking was the only parameter statistically associated with PCR negativity.

Table 1. Demographic and exposure histories for 97 sanitary workers from Spain, comparing statistical differences between Bartonella PCR-positive and PCR-negative individuals.

\begin{tabular}{|c|c|c|c|c|c|}
\hline Demographics and Travel & $\begin{array}{c}\text { PCR+ } \\
n=19(\%)\end{array}$ & $\begin{array}{c}\text { PCR- } \\
\mathrm{n}=78(\%)\end{array}$ & U/OR & $95 \%$ CI & $p$-Values \\
\hline \multicolumn{6}{|l|}{ Age (years) } \\
\hline Median & 35.0 & 39.5 & & & \\
\hline Mean & 36.8 & 41.2 & $\mathrm{U}=527.5$ & & 0.174 \\
\hline Minimum & 21 & 21 & & & \\
\hline Maximum & 55 & 64 & & & \\
\hline \multicolumn{6}{|l|}{ Gender } \\
\hline Female & $13(68.4 \%)$ & $53(67.9 \%)$ & 0.978 & $0.272-3.169$ & 1 \\
\hline Male & $6(31.6 \%)$ & $25(32.1 \%)$ & & & \\
\hline
\end{tabular}


Table 1. Cont.

\begin{tabular}{|c|c|c|c|c|c|}
\hline Demographics and Travel & $\begin{array}{c}\text { PCR+ } \\
\mathrm{n}=19(\%)\end{array}$ & $\begin{array}{c}\text { PCR- } \\
\mathrm{n}=78(\%)\end{array}$ & U/OR & $95 \% \mathrm{CI}$ & $p$-Values \\
\hline \multicolumn{6}{|l|}{ Housing } \\
\hline Urban & $15(78.9 \%)$ & $62(79.5 \%)$ & & & \\
\hline Peri-urban & $4(21.1 \%)$ & $11(14.1 \%)$ & & & 0.798 \\
\hline Rural area/farm & $0(0 \%)$ & $3(3.8 \%)$ & & & \\
\hline Rural area/forest & $0(0 \%)$ & $1(1.3 \%)$ & & & \\
\hline \multicolumn{6}{|l|}{ Clinical condition } \\
\hline Healthy & $17(89.5 \%)$ & $66(84.6 \%)$ & 1.539 & $0.297-15.463$ & 0.731 \\
\hline Persistent/chronic disease & $1(5.3 \%)$ & $13(16.7 \%)$ & 0.280 & $0.006-2.118$ & 0.291 \\
\hline Infectious disease & $2(10.5 \%)$ & $8(10.3 \%)$ & 1.029 & $0.098-5.852$ & 1 \\
\hline \multicolumn{6}{|l|}{ Clinical features } \\
\hline Persistent fever & $0(0 \%)$ & $3(3.8 \%)$ & 0 & $0-10.152$ & 1 \\
\hline Fatigue & $2(10.5 \%)$ & $4(5.1 \%)$ & 2.156 & $0.181-16.520$ & 0.334 \\
\hline Insomnia & $0(0 \%)$ & $8(10.3 \%)$ & 0 & $0-2.388$ & 0.349 \\
\hline Sleepiness & $1(5.3 \%)$ & $1(1.3 \%)$ & 4.191 & $0.052-339.02$ & 0.355 \\
\hline Memory problems & $0(0 \%)$ & $1(1.3 \%)$ & 0 & 0-159.708 & 1 \\
\hline Headache & $3(15.8 \%)$ & $16(20.5 \%)$ & 0.729 & $0.121-3.029$ & 0.758 \\
\hline Irritability & $0(0 \%)$ & $3(3.8 \%)$ & 0 & 0-10.152 & 1 \\
\hline Anxiety & $0(0 \%)$ & $2(2.6 \%)$ & 0 & $0-22.196$ & 1 \\
\hline Depression & $0(0 \%)$ & $0(0 \%)$ & - & - & - \\
\hline Tremor & $0(0 \%)$ & $2(2.6 \%)$ & 0 & $0-22.196$ & 1 \\
\hline Vision impairment & $0(0 \%)$ & $0(0 \%)$ & - & - & - \\
\hline Eye pain & $0(0 \%)$ & $2(2.6 \%)$ & 0 & $0-22.196$ & 1 \\
\hline Balance problems & $0(0 \%)$ & $1(1.3 \%)$ & 0 & $0-159.708$ & 1 \\
\hline Bladder dysfunction & $0(0 \%)$ & $1(1.3 \%)$ & 0 & $0-159.708$ & 1 \\
\hline Shortness of breath & $0(0 \%)$ & $1(1.3 \%)$ & 0 & $0-159.708$ & 1 \\
\hline Tachycardia & $1(5.3 \%)$ & $2(2.6 \%)$ & 2.092 & $0.034-42.27$ & 0.484 \\
\hline Poor appetite & $0(0 \%)$ & $2(2.6 \%)$ & 0 & 0-22.196 & 1 \\
\hline Weight gain & $1(5.3 \%)$ & $3(3.8 \%)$ & 1.384 & $0.025-18.428$ & 1 \\
\hline Chronic diarrhea & $0(0 \%)$ & $1(1.3 \%)$ & 0 & 0-159.708 & 1 \\
\hline Corticosteroid treatment & $2(10.5 \%)$ & $5(6.4 \%)$ & 1.707 & $0.150-11.573$ & 0.620 \\
\hline Allergy & $3(15.8 \%)$ & $26(33.3 \%)$ & 0.378 & $0.065-1.498$ & 0.169 \\
\hline Autoimmune disease & $0(0 \%)$ & $1(1.3 \%)$ & 0 & $0-159.708$ & 1 \\
\hline Dried fruits & $0(0 \%)$ & $2(2.6 \%)$ & 0 & $0-22.196$ & 1 \\
\hline Metals & $0(0 \%)$ & $3(3.8 \%)$ & 0 & $0-10.152$ & 1 \\
\hline Food & $0(0 \%)$ & $0(0 \%)$ & - & - & - \\
\hline Animals & $2(10.5 \%)$ & $1(1.3 \%)$ & 8.762 & $0.433-538.98$ & 0.097 \\
\hline Lactose & $0(0 \%)$ & $0(0 \%)$ & - & - & - \\
\hline Mites & $1(5.3 \%)$ & $4(5.1 \%)$ & 1.027 & $0.020-11.243$ & 1 \\
\hline Pollen & $1(5.3 \%)$ & $12(15.4 \%)$ & 0.308 & $0.007-2.358$ & 0.453 \\
\hline Pets & $13(68.4 \%)$ & $50(64.1 \%)$ & 1.211 & $0.377-4.333$ & 0.794 \\
\hline Dogs & $10(52.6 \%)$ & $35(44.9 \%)$ & 1.361 & $0.442-4.252$ & 0.613 \\
\hline Cats & $6(31.6 \%)$ & $26(33.3 \%)$ & 0.924 & $0.257-2.983$ & 1 \\
\hline Birds & $3(15.8 \%)$ & $21(26.9 \%)$ & 0.512 & $0.087-2.063$ & 0.387 \\
\hline \multicolumn{6}{|l|}{ Arthropod exposure } \\
\hline Fleas & $4(21.1 \%)$ & $32(41.0 \%)$ & 0.387 & $0.085-1.367$ & 0.121 \\
\hline Ticks & $4(21.1 \%)$ & $29(37.2 \%)$ & 0.454 & $0.100-1.612$ & 0.280 \\
\hline Lice & $3(15.8 \%)$ & $20(25.6 \%)$ & 0.547 & $0.093-2.213$ & 0.549 \\
\hline Bed bugs & $0(0 \%)$ & $5(6.4 \%)$ & 0 & $0-4.559$ & 0.580 \\
\hline \multicolumn{6}{|l|}{ Animal exposure } \\
\hline Dogs & $4(21.1 \%)$ & $17(21.8 \%)$ & 0.957 & $0.204-3.571$ & 1 \\
\hline Cats & $3(15.8 \%)$ & $10(12.8 \%)$ & 1.272 & $0.202-2.756$ & 0.715 \\
\hline \multicolumn{6}{|l|}{ Animal scratches and/or } \\
\hline bites & $2(10.5 \%)$ & $15(19.2 \%)$ & 0.497 & $0.050-2.488$ & 0.511 \\
\hline Dogs & $4(21.1 \%)$ & $17(22.8 \%)$ & 0.957 & $0.204-3.571$ & 1 \\
\hline $\begin{array}{l}\text { Cats } \\
\text { Birds }\end{array}$ & $1(5.3 \%)$ & $5(6.4 \%)$ & 0.813 & $0.016-7.945$ & 1 \\
\hline \multicolumn{6}{|l|}{ Outdoors activities } \\
\hline Trekking & $5(26.3 \%)$ & $44(56.4 \%)$ & 0.280 & $0.072-0.924$ & 0.022 \\
\hline Hunting & 0 & 0 & - & - & - \\
\hline Fishing & $0(0 \%)$ & $3(3.8 \%)$ & 0 & $0-10.152$ & 1 \\
\hline Agriculture & $0(0 \%)$ & $8(10.3 \%)$ & 0 & $0-2.388$ & 0.349 \\
\hline Gardening & $2(10.5 \%)$ & $19(24.4 \%)$ & 0.368 & $0.038-1.792$ & 0.231 \\
\hline \multicolumn{6}{|l|}{ Travel out of Spain } \\
\hline Other European countries & $15(78.9 \%)$ & $66(84.6 \%)$ & 0.685 & $0.173-3.319$ & 0.510 \\
\hline North America & $8(42.1 \%)$ & $28(35.9 \%)$ & 1.295 & $0.401-4.030$ & 0.609 \\
\hline Central America & $7(36.8 \%)$ & $28(35.9 \%)$ & 1.041 & $0.310-3.270$ & 1 \\
\hline South America & $2(10.5 \%)$ & $16(20.5 \%)$ & 0.459 & $0.047-2.277$ & 0.512 \\
\hline Asia & $4(21.1 \%)$ & $13(16.7 \%)$ & 1.329 & $0.277-5.167$ & 0.737 \\
\hline Africa & $4(21.1 \%)$ & $8(10.3 \%)$ & 2.309 & $0.450-10.081$ & 0.243 \\
\hline Australia/New Zealand & $0(0 \%)$ & $5(6.4 \%)$ & 0 & $0-4.559$ & 0.580 \\
\hline
\end{tabular}

Percentages may not total $100 \%$ if participants checked more than one category. U is statistic of the Mann-Whitney test. OR is the odds ratio. Percentages are relative to the group (PCR+ or PCR-). 


\subsection{Bartonella Seroprevalence and Blood Stream Infection}

Serology

Seroreactivity among the six Bartonella spp. or genotypes ranged from $16.5 \%$ to $62.9 \%$ with the lowest percentage reactivity to B. quintana (Table 2). Only 16 of $97(16.5 \%)$ participants were not seroreactive to any test antigen. Seroreactivity to $B$. henselae, B. vinsonii subsp. berkhoffii genotypes I, II and III, and B. koehlerae ranged from $20.6 \%$ to $62.9 \%$. Among individual study participants, seroreactivity patterns varied among the six Bartonella spp. or genotypes used for immunofluorescence assay (IFA) testing (Table 2). All but three Bartonella spp. bacteremic individuals were seroreactive to at least one test antigen. Bartonella spp. IFA seroreactivity was not statistically associated with any specific symptom.

Table 2. Immunofluorescent antibody (IFA) titers to six Bartonella spp. or genotypes for 97 sanitary workers tested for Bartonella exposure. Numerical values represent the number of titers at various dilutions and the total number of seroreactors to each antigen.

\begin{tabular}{|c|c|c|c|c|c|c|}
\hline \multirow[b]{2}{*}{ IFA Titer } & \multicolumn{6}{|c|}{ Bartonella Antigen } \\
\hline & Bh SA2 & $B q$ & $B v b \mathrm{TI}$ & $B v b$ TII & $B v b$ TIII & $B k$ \\
\hline$<64$ & 45 & 81 & 77 & 36 & 42 & 51 \\
\hline 64 & 18 & 11 & 6 & 26 & 27 & 26 \\
\hline 128 & 18 & 4 & 10 & 23 & 15 & 13 \\
\hline 256 & 12 & 1 & 4 & 11 & 12 & 5 \\
\hline 512 or 1024 & 4 & 0 & 0 & 1 & 1 & 2 \\
\hline $\begin{array}{c}* \text { Seroreactive } \\
\%\end{array}$ & $\begin{array}{c}52 \\
53.60 \%\end{array}$ & $\begin{array}{c}16 \\
16.50 \%\end{array}$ & $\begin{array}{c}20 \\
20.60 \%\end{array}$ & $\begin{array}{c}61 \\
62.90 \%\end{array}$ & $\begin{array}{c}55 \\
56.70 \%\end{array}$ & $\begin{array}{c}46 \\
47.40 \%\end{array}$ \\
\hline
\end{tabular}

Bh SA2: Bartonella henselae San Antonio 2 strain; Bq: Bartonella quintana; Bvb TI: Bartonella vinsonii subsp. berkhoffii genotype I; Bvb TII: Bartonella vinsonii subsp. berkhoffii genotype II; Bvb TIII: Bartonella vinsonii subsp. berkhoffii genotype III; Bk: Bartonella koehlerae. * Total seroreactive: number and \% of individuals with titers $\geq 64$.

\subsection{BAPGM Enrichment Blood Culture PCR}

Twenty-one participants (21.6\%) had a positive Bartonella alpha-Proteobacteria growth medium (BAPGM) enrichment blood culture/PCR result (Table 3). Bartonella henselae, B. vinsonii subsp. berkhoffii genotype I and B. vinsonii subsp. berkhoffii genotype III DNA was amplified and sequenced directly from extracted blood DNA in four, three, and two individuals, respectively (Table 4). An additional individual was also positive for Bartonella DNA amplification from blood, but species identification was not possible. No direct evidence (PCR/DNA sequencing) of Bartonella vinsonii subsp. berkhoffii genotype II, B. quintana or B. koehlerae was found among participant blood DNA extractions. Following BAPGM enrichment blood culture, sixteen participants were positive for Bartonella DNA amplification or bacterial isolation. Four of nine participants, who were PCR positive following blood DNA extraction (three $B$. vinsonii subsp. berkhoffii genotype I, and a single B. henselae), were again PCR/DNA sequence positive for the same organism following BAPGM enrichment blood culture. The species identified in blood cultures were B. henselae (six participants), B. vinsonii subsp. berkhoffii genotype I (six participants), B. vinsonii subsp. berkhoffii genotype III (four participants), and B. quintana (one participant) (Table 4). Co-infections with B. quintana and B. vinsonii subsp. berkhoffii genotype III, or B. henselae and B. vinsonii subsp. berkhoffii genotype III were each detected in a single participant. Bartonella quintana and $B$. henselae blood agar plate isolates were obtained from two and one participant, respectively (Table 3). PCR sequencing results for the 16S-23S ITS region, groEl, rpoB and 16S rRNA genes for each isolate are provided in Table 4. 
Table 3. Blood and Bartonella alpha-Proteobacteria growth medium (BAPGM) enrichment blood culture PCR/DNA sequencing results for 97 sanitary workers from Spain.

\begin{tabular}{|c|c|c|c|c|c|}
\hline Participants $(n=97)$ & & Specie & Strain & & \\
\hline Sample Type & Bh SA2 & $B v b \mathrm{TI}$ & $B v b$ TIII & $B q$ & $\begin{array}{c}\text { Any } \\
\text { Species }\end{array}$ \\
\hline Blood & 4 & 3 & 2 & 0 & 9 \\
\hline 7-day culture & 0 & 2 & 4 & 0 & 6 \\
\hline 14-day culture & 3 & 1 & 0 & 0 & 4 \\
\hline 21-day culture & 3 & 3 & 0 & 1 & 7 \\
\hline Agar plate isolates & 1 & 0 & 0 & 2 & 3 \\
\hline * Total positive participants (\%) & $9(9.3 \%)$ & $6(6.2 \%)$ & $6(5.2 \%)$ & $2(2.1 \%)$ & $21(21.6 \%)$ \\
\hline
\end{tabular}

Bh: Bartonella henselae San Antonio 2 strain; Bvb TI: Bartonella vinsonii subsp. berkhoffii genotype I; Bvb TIII: Bartonella vinsonii subsp. berkhoffii genotype III; Bq: Bartonella quintana. * Total \% bacteremic.

Table 4. Sequence identity comparisons for the three Bartonella agar plate isolates obtained following subculture.

\begin{tabular}{|c|c|c|c|c|c|c|c|c|c|}
\hline \multirow{2}{*}{ Isolate } & \multirow{2}{*}{$\begin{array}{l}\text { Bacterial } \\
\text { Species }\end{array}$} & \multicolumn{2}{|c|}{ ITS Region } & \multicolumn{2}{|c|}{ GroEl Gene } & \multicolumn{2}{|c|}{ rpoB Gene } & \multicolumn{2}{|c|}{ 16S rRNA } \\
\hline & & $\begin{array}{c}\text { GenBank } \\
\text { ID }\end{array}$ & bp (\%) & $\begin{array}{c}\text { GenBank } \\
\text { ID }\end{array}$ & bp (\%) & $\begin{array}{l}\text { GenBank } \\
\text { ID }\end{array}$ & bp (\%) & $\begin{array}{c}\text { GenBank } \\
\text { ID }\end{array}$ & bp (\%) \\
\hline $\begin{array}{c}\text { GL-90 } \\
B q\end{array}$ & $B q$ Toulouse & BX897700 & $\begin{array}{c}541 / 541 \\
100\end{array}$ & BX897700 & $\begin{array}{c}525 / 525 \\
100\end{array}$ & BX897700 & $\begin{array}{c}593 / 593 \\
100\end{array}$ & BX897700 & $\begin{array}{c}870 / 870 \\
100\end{array}$ \\
\hline $\begin{array}{c}\text { GL-92 } \\
B q\end{array}$ & $B q$ Toulouse & BX897700 & $\begin{array}{c}559 / 559 \\
100\end{array}$ & BX897700 & $\begin{array}{c}525 / 525 \\
100\end{array}$ & BX897700 & $\begin{array}{c}593 / 593 \\
100\end{array}$ & BX897700 & $\begin{array}{c}877 / 877 \\
100\end{array}$ \\
\hline \multirow[t]{2}{*}{$\begin{array}{c}\text { GL-96 } \\
\text { Bh }\end{array}$} & Bh Houston I & СР020742 & $\begin{array}{c}507 / 536 \\
94.7\end{array}$ & СР020742 & $\begin{array}{c}519 / 519 \\
100\end{array}$ & AF171070 & $\begin{array}{c}600 / 600 \\
100\end{array}$ & СР020742 & $\begin{array}{c}878 / 878 \\
100\end{array}$ \\
\hline & Bh SA2 & AF369529 & $\begin{array}{c}536 / 536 \\
100\end{array}$ & AF304021 & $\begin{array}{c}482 / 482 * \\
100\end{array}$ & $\mathrm{NA}^{*}$ & & $\mathrm{NA}^{*}$ & \\
\hline
\end{tabular}

bp: base pairs; Bq: Bartonella quintana; Bh: Bartonella henselae. ${ }^{*}$ NA partial or no comparable sequence data in GenBank for B. henselae San Antonio 2 (SA2) strain.

\subsection{Assay Associations (Serology and BAPGM Enrichment Blood Culture-PCR)}

Seventeen of 21 bacteremic individuals were seroreactive to at least one Bartonella sp. antigen (three seronegative participants were PCR positive in blood culture and/or by bacterial isolation). Interestingly, two $B$. henselae bacteremic patients were not $B$. henselae seroreactive, but one each was seroreactive to $B$. vinsonii subsp. berkhoffii genotypes II and III, or B. vinsonii subsp. berkhoffii genotype II and B. koehlerae (Table 5). BAPGM enrichment blood culture/PCR positivity was not associated with self-reported symptoms. Corticosteroid intake, demographics, and arthropod or animal exposures were not statistically associated with BAPGM enrichment blood culture/PCR positivity (Table 1). Information about exposures, demographics, serologic assays and clinical features of the individuals with BAPGM enrichment blood culture/PCR positivity are summarized in Table 6. 
Table 5. IFA serology results for sanitary workers that were Bartonella PCR positive.

\begin{tabular}{|c|c|c|c|c|c|c|c|}
\hline \multicolumn{2}{|c|}{$\begin{array}{c}\text { Sanitary Worker ID/Bartonella spp. or } \\
\text { Genotype Sequenced }\end{array}$} & \multicolumn{6}{|c|}{ IFA titers } \\
\hline ID & $\begin{array}{l}\text { Bartonella DNA PCR } \\
\text { Species/Genotype }\end{array}$ & $B h$ & $B q$ & $B v b \mathrm{TI}$ & $\begin{array}{c}B v b \\
\text { TII }\end{array}$ & $\begin{array}{l}B v b \\
\text { TIII }\end{array}$ & $B k$ \\
\hline GL-6 & Bvb TIII & 256 & 128 & 128 & 256 & 256 & 128 \\
\hline GL-17 & $B h$ & $<16$ & $<16$ & 32 & 64 & 64 & $<16$ \\
\hline GL-18 & $B h$ & 256 & 64 & 32 & 64 & 128 & 64 \\
\hline GL-21 & $B h$ & 512 & $<16$ & $<16$ & 32 & 32 & 16 \\
\hline GL-26 & $B h$ & 128 & 16 & $<16$ & 16 & 16 & $<16$ \\
\hline GL-32 & $B v b$ TIII $+B h$ & 512 & $<16$ & $<16$ & $<16$ & 64 & 128 \\
\hline GL-37 & $B v b$ TI & 64 & 256 & 128 & 256 & 64 & 128 \\
\hline GL-47 & Bvb TIII & $<16$ & $<16$ & $<16$ & 32 & 32 & 32 \\
\hline GL-49 & $B v b \mathrm{TI}$ & 64 & 16 & 16 & 64 & 256 & 64 \\
\hline GL-58 & $B v b \mathrm{TI}$ & 32 & $<16$ & 32 & 256 & 256 & 1024 \\
\hline GL-65 & $B h$ & 16 & $<16$ & $<16$ & 64 & 32 & 64 \\
\hline GL-80 & $B v b \mathrm{TI}$ & 256 & 128 & 256 & 512 & 256 & 256 \\
\hline GL-84 & $B h$ & 256 & 128 & 256 & 256 & 256 & 256 \\
\hline GL-86 & $B v b \mathrm{TI}$ & $<16$ & $<16$ & 32 & 64 & 32 & 32 \\
\hline GL-87 & $B h$ & 128 & 64 & 128 & 128 & 64 & 64 \\
\hline GL-90 & $B q$ & 32 & $<16$ & $<16$ & $<16$ & 16 & $<16$ \\
\hline GL-92 & $B v b \mathrm{TIII}+B q$ & $<16$ & $<16$ & $<16$ & $<16$ & 16 & 32 \\
\hline GL-96 & $B h$ & 256 & 64 & 256 & 256 & 128 & 128 \\
\hline GL-98 & $B v b$ TIII & $<16$ & $<16$ & 16 & 32 & 64 & $<16$ \\
\hline GL-100 & $B v b \mathrm{TI}$ & 64 & $<16$ & 128 & 256 & 64 & 64 \\
\hline
\end{tabular}

ID: identification number; Bh: Bartonella henselae; Bq: Bartonella quintana; Bvb TI: Bartonella vinsonii subsp. berkhoffii genotype I; Bvb TII: Bartonella vinsonii subsp. berkhoffii genotype II; Bvb TIII: Bartonella vinsonii subsp. berkhoffii genotype III; Bk: Bartonella koehlerae. 
Table 6. Demographics, health status and exposures of sanitary workers with BAPGM enrichment blood culture/PCR positivity.

\begin{tabular}{|c|c|c|c|c|c|c|c|c|c|c|}
\hline $\begin{array}{c}\text { Bartonella spp. } \\
\text { by BAPGM + PCR }\end{array}$ & Age & Sex & $\begin{array}{l}\text { Health } \\
\text { Status }\end{array}$ & $\begin{array}{c}\text { Bartonella } \\
\text { IFA } \\
\text { Sero-Reactivity }\end{array}$ & $\begin{array}{l}\text { Living } \\
\text { Area }\end{array}$ & $\begin{array}{c}\text { Cat } \\
\text { Exposure }\end{array}$ & $\begin{array}{c}\text { Dog } \\
\text { Exposure }\end{array}$ & $\begin{array}{l}\text { Other Animal } \\
\text { Exposure }\end{array}$ & Outdoor Activities & Arthropod Exposure \\
\hline $\begin{array}{c}\text { Bvb TIII } \\
6\end{array}$ & 34 & F & Healthy & $\begin{array}{c}\text { Pos } \\
B v b, B q, B h, B k\end{array}$ & Urban & $\begin{array}{l}\text { Yes } \\
\text { (bite) }\end{array}$ & $\begin{array}{l}\text { Yes } \\
\text { (bite) }\end{array}$ & Rodent & Gardening & No \\
\hline $\begin{array}{l}\boldsymbol{B} h \\
17\end{array}$ & 40 & M & Healthy & $\begin{array}{l}\text { Pos } \\
B v b\end{array}$ & Urban & No & Yes & No & $\begin{array}{l}\text { Trekking Cycling } \\
\text { Diving }\end{array}$ & $\begin{array}{c}\text { Fleas-Ticks-Biting } \\
\text { flies-Mosquitoes-Spiders }\end{array}$ \\
\hline $\begin{array}{l}B h \\
18\end{array}$ & 43 & $\mathrm{~F}$ & Healthy & $\begin{array}{c}\text { Pos } \\
B v b, B q, B h, B k\end{array}$ & Urban & Yes & No & Rodent & No & No \\
\hline $\begin{array}{l}\text { Bh } \\
21\end{array}$ & 39 & M & Healthy & $\begin{array}{l}\text { Pos } \\
B h\end{array}$ & Urban & Yes & No & $\begin{array}{c}\text { Bird } \\
\text { Reptile } \\
\text { Hedgehog }\end{array}$ & No & Fleas-Ticks-Mosquitoes-Spiders \\
\hline $\begin{array}{l}B h \\
26\end{array}$ & 31 & $\mathrm{~F}$ & Healthy & $\begin{array}{c}\text { Pos } \\
B h\end{array}$ & Urban & Yes & No & No & Trekking & Fleas-Ticks-Spiders \\
\hline $\begin{array}{c}B v b \mathrm{TIII}+B h \\
32\end{array}$ & 33 & $\mathrm{~F}$ & Healthy & $\begin{array}{c}\text { Pos } \\
B v b, B h, B k\end{array}$ & Peri-urban & Yes & Yes & Poultry & No & Mosquitoes \\
\hline $\begin{array}{c}B v b \text { TI } \\
37\end{array}$ & 21 & $\mathrm{~F}$ & Healthy & $\begin{array}{c}\text { Pos } \\
B v b, B q, B h, B k\end{array}$ & Urban & No & Yes & No & No & No \\
\hline $\begin{array}{c}B v b \text { TIII } \\
\quad 47 \\
\end{array}$ & 33 & M & Healthy & Neg & Urban & No & No & No & Cycling & No \\
\hline $\begin{array}{c}\text { Bvb TI } \\
49\end{array}$ & 21 & $\mathrm{~F}$ & Healthy & $\begin{array}{c}\text { Pos } \\
B v b, B h, B k\end{array}$ & Urban & No & No & No & No & No \\
\hline $\begin{array}{l}\text { Bvb TI } \\
58\end{array}$ & 27 & F & Healthy & $\begin{array}{c}\text { Pos } \\
B v b, B k\end{array}$ & Urban & $\begin{array}{c}\text { Yes } \\
\text { (bite) }\end{array}$ & $\begin{array}{c}\text { Yes } \\
\text { (bite) }\end{array}$ & No & Trekking & $\begin{array}{c}\text { Fleas- } \\
\text { Biting flies-Lice-Mites }\end{array}$ \\
\hline $\begin{array}{l}B h \\
65\end{array}$ & N.A. & F & N.A. & $\begin{array}{c}\text { Pos } \\
B v b, B k\end{array}$ & Peri-urban & No & No & No & No & No \\
\hline $\begin{array}{l}B v b \text { TI } \\
80\end{array}$ & 31 & $\mathrm{~F}$ & Healthy & $\begin{array}{c}\text { Pos } \\
B v b, B q, B h, B k\end{array}$ & Urban & No & No & No & Cycling & Mosquitoes \\
\hline $\begin{array}{l}B h \\
84\end{array}$ & 48 & F & Healthy & $\begin{array}{c}\text { Pos } \\
B v b, B q, B h, B k\end{array}$ & Urban & No & No & No & No & No \\
\hline $\begin{array}{c}B v b \text { TI } \\
86\end{array}$ & 36 & M & Healthy & $\begin{array}{l}\text { Pos } \\
B v b\end{array}$ & Urban & No & Yes & No & No & No \\
\hline
\end{tabular}


Table 6. Cont

\begin{tabular}{|c|c|c|c|c|c|c|c|c|c|c|}
\hline $\begin{array}{l}\text { Bartonella spp. } \\
\text { by BAPGM + PCR }\end{array}$ & Age & Sex & $\begin{array}{l}\text { Health } \\
\text { Status }\end{array}$ & $\begin{array}{c}\text { Bartonella } \\
\text { IFA } \\
\text { Sero-Reactivity }\end{array}$ & $\begin{array}{l}\text { Living } \\
\text { Area }\end{array}$ & $\underset{\text { Exposure }}{\text { Cat }}$ & $\begin{array}{c}\text { Dog } \\
\text { Exposure }\end{array}$ & $\begin{array}{l}\text { Other Animal } \\
\text { Exposure }\end{array}$ & Outdoor Activities & Arthropod Exposure \\
\hline $\begin{array}{l}B h \\
87\end{array}$ & 52 & $\mathrm{~F}$ & Healthy & $\begin{array}{c}\text { Pos } \\
B v b, B q, B h, B k\end{array}$ & Urban & No & No & No & No & No \\
\hline $\begin{array}{l}B q \\
90\end{array}$ & 30 & $\mathrm{M}$ & Healthy & $\mathrm{Neg}$ & Urban & $\begin{array}{c}\text { Yes } \\
\text { (bite) }\end{array}$ & Yes & $\begin{array}{c}\text { Bird } \\
\text { Poultry } \\
\text { Pigs } \\
\text { Sheep }\end{array}$ & $\begin{array}{c}\text { Trekking } \\
\text { Cycling Diving }\end{array}$ & $\begin{array}{c}\text { Fleas-Ticks-Biting } \\
\text { flies-Mosquitoes-Lice-Spiders }\end{array}$ \\
\hline $\begin{array}{l}B v b \text { TIII }+B q \\
92\end{array}$ & 38 & $\mathrm{M}$ & Healthy & $\mathrm{Neg}$ & Urban & No & Yes & No & No & Biting flies-Mosquitoes-Spiders \\
\hline $\begin{array}{l}B h \\
96\end{array}$ & 55 & $\mathrm{~F}$ & Healthy & $\begin{array}{c}\text { Pos } \\
B v b, B q, B h, B k\end{array}$ & Peri-urban & No & Yes & No & No & No \\
\hline $\begin{array}{l}B v b \text { TIII } \\
\quad 98\end{array}$ & 43 & $\mathrm{~F}$ & Healthy & $\begin{array}{l}\text { Pos } \\
B v b\end{array}$ & Urban & $\begin{array}{c}\text { Yes } \\
\text { (bite) }\end{array}$ & No & No & Trekking & $\begin{array}{c}\text { Ticks- } \\
\text { Biting } \\
\text { flies-Mosquitoes-Lice-Spiders }\end{array}$ \\
\hline $\begin{array}{l}B v b \text { TI } \\
100\end{array}$ & 50 & $\mathrm{~F}$ & Healthy & $\begin{array}{c}\text { Pos } \\
B v b, B h, B k\end{array}$ & Peri-urban & No & $\begin{array}{c}\text { Yes } \\
\text { (bite) }\end{array}$ & $\begin{array}{l}\text { Bird } \\
\text { Rabbit } \\
\text { Rodent }\end{array}$ & $\begin{array}{l}\text { Trekking } \\
\text { Gardening } \\
\text { Cycling } \\
\text { Diving }\end{array}$ & $\begin{array}{c}\text { Ticks- } \\
\text { Biting flies-Mosquitoes-Lice }\end{array}$ \\
\hline
\end{tabular}

F: female; M: male; Bq: Bartonella quintana; Bvb TIII: Bartonella vinsonii subsp. berkhoffii genotype III; Bh: Bartonella henselae; Bvb TI: Bartonella vinsonii subsp. berkhoffii genotype I; N.A.: Data not available. 


\section{Discussion}

In this study, conducted among 'healthy volunteers' working in a sanitary setting in Spain, $83.1 \%$ participants were Bartonella seroreactive and $21.6 \%$ had molecular evidence of bloodstream infection using the BAPGM enrichment blood culture-PCR platform. These high percentages are unprecedented in the few published studies testing healthy participants against a large (6 Bartonella strains) IFA serology panel, as well as the high prevalence of occult bloodstream infection among individuals from the Rioja region of Spain $[15,16]$. Using the same IFA assays, a previous study of 32 healthy medical personnel from North Carolina identified only one B. henselae seroreactor (antibody titer 64); however, 16 (50\%) individuals were B. vinsonii subsp. berkhoffii genotype II seroreactive [19]. Among these North Carolina healthy individuals, no participant was B. vinsonii subsp. berkhoffii genotype I or III, B. koehlerae, or B. quintana seroreactive and Bartonella spp. DNA was not PCR amplified from any blood or BAPGM enrichment blood culture using the same diagnostic platform used in this study $[19,20]$. Testing of stored blood samples from these 32 individuals over a decade later by droplet digital PCR (ddPCR) also resulted in negative Bartonella spp. DNA results (Maggi R. unpublished data). Technically, the same personnel (Bradley and Maggi) performed the serology and BAPGM enrichment blood culture testing in the current and previous North Carolina study. Based upon the results of this study, Bartonella spp. occupational exposure risk in sanitary workers should be further investigated.

Interestingly, B. vinsonii subsp. berkhoffii genotype II seroreactivity was unexpectedly high among healthy sanitary workers in both studies (62.9\% Spain, and 50\% North Carolina). In the only other study involving healthy individuals and the BAPGM enrichment blood culture platform, Brazilian medical personnel screened 500 blood donors $[15,16]$. Seroprevalence was $16 \%$ and $32 \%$, respectively for B. henselae and B. quintana (IFA antigen slides provided by the Centers for Disease Control and Prevention), and blood stream infection was found in $16(3.2 \%)$ of the 500 donors, infected with either B. henselae $(\mathrm{n}=15)$ or B. clarridgeiae $(\mathrm{n}=1)$. It is important to point out that similar to the current study, IFA serology was not consistently associated with bloodstream infection. Recent documentation of bloodstream infection among healthy individuals has further increased the diagnostic complexity associated with the genus Bartonella. As fastidious Gram-negative bacteria, Bartonella spp. require several weeks to grow axenically. Despite efforts to improve yield, obtaining agar plate isolates remains difficult and successful isolation has only been reported in a small number of human cases, often requiring microbiological techniques limited to specialty laboratories. Isolation is more likely to occur when culturing immunocompromised individuals, who presumably maintain higher levels of bacteremia [21]. Serological assays (IFA) are universally used for diagnosis of Bartonella infection in animals and humans, although sensitivity has been considered poor [22,23]. In the context of IFA specificity, cross reactions with other bacterial pathogens such as Coxiella burnetii, Chlamydia spp., Rickettsia spp. (spotted fever group), Treponema pallidum, Orientia tsutsugamushi, Francisella tularensis, Ehrlichia chaffeensis, Mycoplasma pneumoniae, and Escherichia coli have been reported in human patients $[24,25]$. In context of One Health, Bartonella spp. IFA has a high degree of specificity $(97 \%$ or greater depending upon IFA antigen) when testing dogs [26-28]. Sera from dogs with very high IFA titers (8192) following experimental infection with Rickettsia rickettsii do not induce fluorescence (cross reactivity) to Bartonella spp. antigens [29]. However, similar to humans, Bartonella IFA assays have poor sensitivity when testing sera from PCR/culture positive dogs [30]. The extent to which sequential or simultaneous exposure to multiple Bartonella spp. or the frequency of Bartonella intergenus IFA specificity contributes to serology results in dogs and humans, when using a broad panel of antigens that differ from the classical B. henselae and B. quintana diagnostic assays, awaits additional clarification. Dogs also developed Bartonella species-specific IFA antibodies following experimental infection with $B$. henselae or B. vinsonii subsp. berkhoffii $[26,27]$. Similarly, a veterinarian developed B. vinsonii subsp. berkhoffii genotype-specific antibodies after an inadvertent needle stick transmission [31]. Overall, evidence to support Bartonella spp. IFA cross-reactivity in dogs to closely related alpha-Proteobacteria or other more distantly related bacteria is lacking and the extent to which co-exposures, rather than cross-reactivity, contributes to serology findings in humans remains unclear. For people who live in 
places like La Rioja where free time is often spent in rural environments, co-exposures may be more frequent than previously suspected.

Demonstration of Bartonella spp. DNA in blood or tissues using PCR assays can be technically limiting due to low level infection (minimal target DNA), a relapsing bacteremia, or Bartonella PCR primer designs that differ from the numerous Bartonella spp. genotype and strain targets (failure to anneal and amplify). Thus, negative PCR results do not exclude the presence of authentic Bartonella infections, as previously described [21]. Histological examination (Warthin-Starry staining) of a biopsy specimen at a site of systemic involvement is often not sufficiently specific to establish a diagnosis of Bartonella infection since other infectious diseases (e.g., tularemia) cannot be readily distinguished. Although immunohistochemical analyses are less sensitive than PCR amplification techniques, their usefulness to establish the etiologic diagnosis of Bartonella infections has been reported, but these assays are available in only a few laboratories [32].

Recently, specialized culture techniques based on growth enrichment in modified media combined with PCR assays and subculture bacterial isolation (BAPGM platform) have been developed with the aim of enhancing documentation of Bartonella infection [33,34]. Validation of the BAPGM enrichment blood culture/PCR platform for the assessment of Bartonella spp. bloodstream infection in dogs was originally reported in the Journal of Microbiological Methods [35,36]. Subsequently the BAPGM platform has been used diagnostically to assess bloodstream infection in dogs, other animal species, $[37,38]$ and in humans [19,20,39-41]. It is clear that the developmental and microbiological utilization of more sensitive or robust microbiological techniques such as MALDI TOF, Next Generation Sequencing and specialized enrichment/PCR techniques enhance the detection of microorganisms in various patient samples [42-44].

At times, these more sensitive techniques detect organisms in healthy individuals that were previously associated with pathology in sick individuals, which is seemingly historically applicable to the genus Bartonella. Increased detection sensitivity can be a benefit to the patient and physician, but as illustrated by the results of this study, increased sensitivity can also complicate clinical decision-making, as well as microbiological and pathological interpretations. In this study, statistically significant associations were not detected between serology or the detection of Bartonella bloodstream infection and self-reported symptoms, such as fatigue and insomnia.

In the current study, BAPGM enrichment blood culture/PCR resulted in the amplification of B. vinsonii subsp. berkhoffii genotype III DNA from four participants. In contrast to the results of this study, human studies from the United States using the BAPGM platform most often result in amplification of B. vinsonii subsp. berkhoffii genotype II and less frequently genotype I DNA [19]. To our knowledge, human infection with B. vinsonii genotype III has not been reported from North America, whereas this genotype has been identified in dogs and humans in several Mediterranean countries, including Spain $[18,45,46]$. Interestingly, the genotype III was also documented in military working dogs with endocarditis imported to the United States from Europe [47]. In addition to all PCR negative controls remaining negative throughout the study, failure to detect $B$. vinsonii subsp. berkhoffii genotype II, in conjunction with the detection of genotype III provides further support for a lack of laboratory contamination with PCR products or organisms. In addition, the B. henselae and B. quintana sequences obtained in this study were most consistent with sequences reported by European, as compared to North American investigators (Table 4 and analysis by Maggi RG).

The results of this study have created additional diagnostic challenges for clinicians attempting to confirm a diagnosis of bartonellosis in an individual patient. For example, microbiological criteria for a clinical diagnosis of Bartonella endocarditis in a patient with a blood culture-negative endocarditis (BCNE), include Bartonella spp. PCR positive or an IFA antibody titer of $\geq 800$ against $B$. henselae or B. quintana antigens [48]. Amplification of B. henselae or B. quintana DNA from excised heart valves also establishes a diagnosis of Bartonella endocarditis in BCNE cases $[9,48]$. The prevalence of Bartonella infection using serological techniques is also a cause of concern. In the present study, Bartonella seroprevalence in healthy participants $(>83 \%)$ was substantially higher than the prevalence's 
reported from blood donors from the same area nearly 20 years before $(<6 \%)$, and even exceeded previous seroprevalence values from risk groups, such as cat owners $(28.9 \%)$ and HIV-infected people $(17.3 \%)$ [49,50]. Clearly, differences in the number of Bartonella spp. antigens and variable sensitivity among individual IFA antigens used at these two times are likely contributors, but it is also possible that the epidemiology of Bartonella spp. transmission has changed in the region. In support of this possibility, using the same antigens, the seroprevalence of sanitary workers was more than twice the seroprevalence found among veterinary sanitary workers from different regions of Spain according to a study performed during the same year [18]. The results of this study only marginally impact the clinical interpretation of results for BCNE patients; however, our findings complicate interpretation of serology and PCR results for patients with non-specific symptoms, chronic lymphadenitis, granulomatous hepatitis and other forms of pathology, particularly when Bartonella DNA is amplified, but antibody reactivity is low or not detected. Obviously, a single serological result (an IgG seroreactive value) does not confirm an acute or chronic infection, since IgG antibodies may be due to a prior exposure to the microorganism.

Recent documentation of the historical co-evolutionary efficiency of Bartonella spp. among mammalian reservoir hosts located throughout much of the world represents an amazing and evolving area of research [51,52]. In addition to transmission by a substantial number of documented and suspected vectors, Bartonella spp. have been transmitted by needle stick, animal bites and scratches and potentially by blood transfusion $[31,53]$. Thus, our epidemiological understanding of this genus continues to evolve; thereby influencing medical understanding of transmission patterns among animals and human patients. When bartonellosis is clinically suspected, a diagnostic approach that incorporates results of serology, enrichment culture and molecular techniques should always be interpreted in the context of the medical history, exposure risk and within the differential diagnosis that excludes other microorganisms.

\section{Materials and Methods}

\subsection{Study Design and Subject Recruitment}

A cross-sectional study was performed to determine the seroprevalence to six Bartonella species/genotypes. Bacteremia was concurrently assessed by means of Bartonella alpha-Proteobacteria growth medium (BAPGM) enrichment blood culture platform. Institutional review board approval for this study was obtained from the Ethical Committee of Clinical Research from La Rioja (CEICLAR) in January 29, 2016 (Ref. CEICLAR PI-209).

\subsection{Data and Specimen Collection}

A standardized questionnaire including demographic information, symptoms experienced, domestic and wild animal bites, scratches or exposures, and travel history, was completed. Exposure to, or bites by different arthropods (lice, fleas, ticks, mites, bed bugs and others) was recorded. Approximately $10-12 \mathrm{~mL}$ of blood (5-6 mL in EDTA, 5-6 mL in a serum separator tube) was collected at the time of enrollment. Aseptic blood collection, including chlorhexidine decontamination of the skin, was performed by an experienced nurse. Three participants, who reported antimicrobial use within the last 2 months on the questionnaire, were subsequently excluded from the study.

\subsection{Specimen Processing and Diagnostic Testing}

Refrigerated EDTA-anticoagulated blood and serum samples were processed in less than two hours at the Center of Rickettsiosis and Arthropod-Borne Diseases (CRETAV), located at the Center for Biomedical Research from La Rioja (CIBIR, Logroño, La Rioja, Spain), where blood was centrifuged and sera stored at $-80^{\circ} \mathrm{C}$ until prepared for shipping to Galaxy Diagnostics, Inc., Research Triangle Park, North Carolina, USA. 


\subsection{Bartonella IFA Serological Testing}

Bartonella vinsonii subsp. berkhoffii, B. henselae, B. koehlerae and B. quintana antibodies were determined in the Intracellular Pathogens Research Laboratory (IPRL) at North Carolina State University (North Carolina, USA) using cell culture grown bacteria as antigens and following standard immunofluorescent antibody assay (IFA) techniques [18,19]. Canine isolates of B. vinsonii subsp. berkhoffii genotype I (NCSU 93CO-01 Tumbleweed, ATCC type strain \#51672), B. vinsonii subsp. berkhoffii genotype II (NCSU 95CO-08, Winnie) and B. vinsonii subsp. berkhoffii genotype III (NCSU 06CO-01 Klara), feline isolates of B. henselae SA2 strain (NCSU 95FO-099, Missy) and B. koehlerae (NCSU 09FO-01, Trillium) and B. quintana (NCSU11-MO-01 Monkey origin) were passed from agar plate grown cultures into Bartonella-permissive cell lines, i.e., the DH82 (a canine monocytoid) cell line for strains $B$. henselae SA2, B. quintana, B. vinsonii subsp. berkhoffii I and B. koehlerae and Vero cells (a mammalian fibroblast cell line) for B. vinsonii subsp. berkhoffii II and III to obtain antigens for IFA testing. For each antigen, heavily infected cell cultures were spotted onto 30-well Teflon-coated slides (Cell-Line/Thermo Scientific), air-dried, acetone-fixed, and stored frozen. Fluorescein conjugated goat anti-human IgG (Cappel, ICN) was used to detect bacteria within cells using a fluorescent microscope (Carl Zeiss Microscopy, LLC, Thornwood, NY). Serum samples diluted in a phosphate-buffered saline (PBS) solution containing normal goat serum, Tween-20, and powdered nonfat dry milk to block nonspecific antigen binding sites were first screened at dilutions of 1:16 to 1:64. All sera that were reactive at a reciprocal titer of 64 were further tested with two-fold dilutions out to 1:8192. To avoid confusion with possible nonspecific binding found at low dilutions, a cutoff of 64 was selected as a seroreactive titer.

\subsection{Growth Medium}

Enrichment blood culture was performed at Galaxy Diagnostics Inc., Research Triangle Park, North Carolina, USA, as previously described [18-20]. An aliquot of $1 \mathrm{~mL}$ of EDTA whole blood was inoculated into $10 \mathrm{~mL}$ of BAPGM, after which the cultures were maintained at $35{ }^{\circ} \mathrm{C}$ in a $5 \% \mathrm{CO}_{2}$, water-saturated atmosphere. After 7, 14, and 21-day incubation periods, PCR was performed on each inoculated liquid culture and a $1 \mathrm{~mL}$ aliquot of the enrichment culture was inoculated onto blood agar plates and incubated at $35^{\circ} \mathrm{C}$. Plates were checked for colony formation at 7,14 , and 21 days after plating. An un-inoculated BAPGM culture (negative control) was processed in an identical manner with each group of study participant specimens.

\subsection{Conventional and Real-Time PCR Analysis}

DNA was extracted using standard operating procedures from EDTA anticoagulated blood, enrichment liquid blood cultures incubated for 7, 14, and 21 days, and from blood agar plate colony isolates, if obtained after subculture from BAPGM enriched blood specimens [19]. Bartonella spp. DNA was amplified using primers designed to amplify two distinct consensus sequences in the Bartonella 16S-23S intergenic spacer (ITS) region as described previously with minor modifications [18]. Two sets of oligonucleotides, 325s and 1100as were used as forward and reverse primers for conventional PCR, and primers 325s and 543as were used as forward and reverse primers for quantitative PCR along with TaqMan probe 438 (Table 1). Additionally, as previously reported [18], conventional PCR screening for B. koehlerae was performed using species-specific oligonucleotides Bkoehl-1s and Bkoehl-1125as as forward and reverse primers, respectively (Table 1). Amplification of the ITS region at both genus and species (B. koehlerae) levels were performed in a $25 \mu \mathrm{L}$ final volume reaction containing $12.5 \mu \mathrm{L}$ of either MyTaq HS Red Mix 2X (Bioline) for B. koehlerae conventional PCR or Sso Advanced Universal Probe Supermix (BioRad) for Bartonella genus real-time PCR; $0.2 \mu \mathrm{L}$ of $100 \mu \mathrm{M}$ of each forward and reverse primer (IDT-DNA Technology), $7.3 \mu \mathrm{L}$ of molecular-grade water, and $5 \mu \mathrm{L}$ of DNA from each sample tested. PCR negative controls were prepared using $5 \mu \mathrm{L}$ of $\mathrm{dH}_{2} \mathrm{O}$ (when testing isolates from plates), $5 \mu \mathrm{L}$ of DNA from blood of a healthy dog, or $5 \mu \mathrm{L}$ of DNA extracted from un-inoculated BAPGM-negative controls (when testing BAPGM enrichment cultures). Positive controls for PCR were 
prepared by serial dilution (using dog blood DNA) of genomic DNA from B. henselae (Houston I strain type) down to $0.001 \mathrm{pg} / \mu \mathrm{L}$ (equivalent to 0.5 bacteria $/ \mu \mathrm{L}$ ). Conventional PCR was performed in an Eppendorf Mastercycler EP gradient under the following conditions-a single hot-start cycle at $95^{\circ} \mathrm{C}$ for $3 \mathrm{~min}$ followed by 55 cycles of denaturing at $94^{\circ} \mathrm{C}$ for 15 seconds (s), annealing at $66^{\circ} \mathrm{C}$ for $15 \mathrm{~s}$, and extension at $72{ }^{\circ} \mathrm{C}$ for $18 \mathrm{~s}$. Amplification was completed by an additional cycle at $72{ }^{\circ} \mathrm{C}$ for $1 \mathrm{~min}$, and products were analyzed by $2 \%$ agarose gel electrophoresis with detection using ethidium bromide under ultraviolet light. Amplicon products were sequenced to determine the Bartonella species and ITS strain type. Real-time PCR was performed in an CFX96 Real-time System (Bio-Rad) under the following conditions: a single hot-start cycle at $95^{\circ} \mathrm{C}$ for $3 \mathrm{~min}$ followed by 44 cycles of denaturing at $94{ }^{\circ} \mathrm{C}$ for $10 \mathrm{~s}$, annealing at $66^{\circ} \mathrm{C}$ for $10 \mathrm{~s}$, and extension at $72{ }^{\circ} \mathrm{C}$ for $10 \mathrm{~s}$. Fluorescence at channel 1 was detected during the extension cycle. As in conventional PCR, all amplicon products were sequenced to determine the Bartonella species and ITS strain type. All PCR and uninoculated BAPGM enrichment controls remained negative throughout the study period. PCR assays targeting GroEl, rpoB and $16 \mathrm{~S}$ rRNA genes were also performed to confirm identification of agar plate isolates (Table 7).

Table 7. Primers and probe used for PCR testing in this study.

\begin{tabular}{ccr}
\hline Oligonucleotide & Type & \multicolumn{1}{c}{ Sequence } \\
\hline BsppITS325s & Sense primer & 5' CCTCAGATGATGATCCCAAGCCTTCTGGCG 3' \\
\hline BsppITS543as & Antisense primer & $5^{\prime}$ AATTGGTGGGCCTGGGAGGACTTG 3' \\
\hline BsppITS1100as & Antisense primer & $5^{\prime}$ GAACCGACGACCCCCTGCTTGCAAAGCA 3' \\
\hline BsppITS438 & TaqMan probe & 5' FAM-AGGTTTTCC/ZEN/GGTTTATCCCGGAGGGC-IABkFQ 3' $^{\prime}$ \\
\hline Bkoehl-1s & Sense primer & $5^{\prime}$ CTTCTAAAATATCGCTTCTAAAAATTGGCATGC 3' \\
\hline Bkoehl1125as & Antisense primer & \\
\hline Sequencing analysis & \\
\hline
\end{tabular}

PCR amplicon DNA sequencing was performed by a commercial company (Genewiz, Research. Triangle Park, NC). Chromatogram evaluation and sequence alignments were performed using ContigExpress and AlignX software (Vector NTI Suite 10.1, Invitrogen Corp., Carlsbad, CA). Bacteria species and genotype were defined by comparing similarities with other sequences deposited in the GenBank database using the Basic Local Alignment Search Tool (Blast v. 2.0), and an in-house curated database (Align X, Vector-NTI-Invitrogen).

\subsection{Data Analysis}

Questionnaire data for the study were collected on paper forms and entered into a Microsoft Excel database. Data entry was validated by comparison of the electronic records with the information on the forms. Associations of demographic, risk factor, symptoms and exposure variables were assessed with means and medians for continuous variables, and with counts and rates in contingency tables for categorical data. Group comparisons were performed. The Fisher exact test for categorical variables and the Mann-Whitney U test for non-categorical variables were used. Data processing was carried out with the R software [18], version 3.3.1 for Windows.

Author Contributions: Conceptualization, A.P., R.M., J.A.O., L.G.-Á., X.R. and E.B.; formal analysis, M.S.-M.; funding acquisition, A.P. and E.B.; investigation, R.M., J.A.O., J.B., L.G.-Á. and X.R.; methodology, R.M. and J.B.; project administration, E.B.; resources, A.P., R.M. and E.B.; supervision, J.A.O. and E.B.; validation, A.P., R.M., J.A.O. and E.B.; writing-original draft, A.P., R.M., J.A.O., J.B. and E.B.; writing-review and editing, A.P., R.M., J.B., X.R. and E.B. All authors read and approved the final manuscript.

Funding: This research was funded by Bayer Animal Health GmbH (Leverkusen, Germany), grant number 2111116219.

Conflicts of Interest: In conjunction with Dr. S. Sontakke and North Carolina State University, E. B. Breitschwerdt holds US Patent No. 7,115,385; Media and Methods for Cultivation of Microorganisms, which was issued on October 3rd, 2006. He is a co-founder, shareholder and Chief Scientific Officer for Galaxy Diagnostics, a company that provides advanced diagnostic testing for the detection of Bartonella spp. infections. The remaining authors declare no conflicts of interest. The funders had no role in the design of the study; in the collection, analyses, or interpretation of data; in the writing of the manuscript, or in the decision to publish the results. This study was presented in part at the International Congress on Rickettsia and other Intracellular Bacteria (ESCCAR meeting), Marseille, France, June 19-21, 2017 (oral presentation), at the 12th Symposium of the Canine Vector Borne Disease 
(CVBD) World Forum, Athens, Greece, March 13-16, 2017 (oral presentation), and at the XXI Congreso de la Sociedad Española de Enfermedades Infecciosas y Microbiología Clínica (SEIMC), Málaga (Spain), May 11-13, 2017 (oral presentations 071 and 072). The funders had no role in the design of the study; in the collection, analyses, or interpretation of data; in the writing of the manuscript, or in the decision to publish the results.

Availability of Data and Materials: Data supporting the conclusions of this article are included in the article. To assure participant confidentiality, please contact JAO or EB for questions relative to the raw data.

Ethics Approval and Consent to Participate: Institutional review board approval for this study was received from the Ethical Committee of Clinical Research from La Rioja (CEICLAR) on January 29, 2016 (Ref. CEICLAR PI-209).

\section{References}

1. Gandhi, T.N.; Slater, L.N.; Welch, D.F.; Koehler, J.E. Bartonella, including cat-scratch disease. In Mandell, Douglas, and Bennett's Principles and Practice of Infectious Diseases, 8th ed.; Bennett, J.E., Dolin, R., Blaser, M.J., Eds.; Elsevier Saunders: Philadelphia, PA, USA, 2015.

2. Battistini, T.S. Estudios Sobre la Verruga Peruana; La Acción Médica: Lima, Peru, 1929.

3. Telford, S.R.; Wormser, G.P. Bartonella spp. transmission by ticks not established. Emerg. Infect. Dis. 2010, 16, 379-384. [CrossRef] [PubMed]

4. Bonilla, D.L.; Kabeya, H.; Henn, J.; Kramer, V.L.; Kosoy, M.Y. Bartonella quintana in body lice and head lice from homeless persons, San Francisco, California, USA. Emerg. Infect. Dis. 2009, 15, 912-915. [CrossRef] [PubMed]

5. Angelakis, E.; Socolovschi, C.; Raoult, D. Bartonella quintana in Cimex hemipterus, Rwanda. Am. J. Trop. Med. Hyg. 2013, 89, 986-987. [CrossRef] [PubMed]

6. Bai, Y.; Osinubi, M.O.V.; Osikowicz, L.; McKee, C.; Vora, N.M.; Rizzo, M.R.; Recuenco, S.; Davis, L.; Niezgoda, M.; Ehimiyein, A.M.; et al. Idanre Bat Festival Investigation Team. Human exposure to novel Bartonella species from contact with fruit bats. Emerg. Infect. Dis. 2018, 24, 2317-2323. [CrossRef] [PubMed]

7. Kabeya, H.; Colborn, J.M.; Bai, Y.; Lerdthusnee, K.; Richardson, J.H.; Maruyama, S.; Kosoy, M.Y. Detection of Bartonella tamiae DNA in ectoparasites from rodents in Thailand and their sequence similarity with bacterial cultures from Thai patients. Vector Borne Zoonotic Dis. 2010, 10, 429-434. [CrossRef]

8. Blanco, J.R.; Oteo, J.A. Rochalimaea spp. infections and associated diseases. Med. Clin. 1996, 107, 593-596.

9. Oteo, J.A.; Castilla, A.; Arosey, A.; Blanco, J.R.; Ibarra, V.; Morano, L.E. Endocarditis due to Bartonella spp. Three new clinical cases and Spanish literature review. Enferm. Infecc. Microbiol. Clin. 2006, 24, $297-301$. [CrossRef]

10. Zellali, K.; Benard, E.; Smokvina, E.; Belgaid, A.; Labbé, F.; Bertrand, V. Multifocal pelvic osteomyelitis in a child associated with cat-scratch disease: A case report and review of the literature. Paediatr. Int. Child. Health 2019, 39, 290-293. [CrossRef]

11. VanderHeyden, T.R.; Yong, S.L.; Breitschwerdt, E.B.; Maggi, R.G.; Mihalik, A.R.; Parada, J.P.; Fimmel, C.J. Granulomatous hepatitis due to Bartonella henselae infection in an immunocompetent patient. BMC Infect. Dis. 2012, 12, 17. [CrossRef]

12. García, J.C.; Núñez, M.J.; Castro, B.; Fernández, J.M.; López, A.; Portillo, A.; Oteo, J.A. Hepatosplenic cat scratch disease in immunocompetent adults: Report of 3 cases and review of the literature. Medicine 2014, 93, 267-279. [CrossRef]

13. Breitschwerdt, E.B. Bartonellosis, one health and all creatures great and small. Vet. Dermatol. 2017, $28,96$. [CrossRef] [PubMed]

14. Breitschwerdt, E.B.; Maggi, R.G. Bartonella quintana and Bartonella vinsonii subsp. vinsonii bloodstream co-infection in a girl from North Carolina, USA. Med. Microbiol. Immunol. 2019, 208, 101-107. [CrossRef] [PubMed]

15. Pitassi, L.H.; de Paiva Diniz, P.P.; Scorpio, D.G.; Drummond, M.R.; Lania, B.G.; Barjas-Castro, M.L.; Gilioli, R.; Colombo, S.; Sowy, S.; Breitschwerdt, E.B.; et al. Bartonella spp. bacteremia in blood donors from Campinas, Brazil. PLoS Negl. Trop. Dis. 2015, 9, e0003467. [CrossRef] [PubMed]

16. Vieira-Damiani, G.; Diniz, P.P.; Pitassi, L.H.; Sowy, S.; Scorpio, D.G.; Lania, B.G.; Drummond, M.R.; Soares, T.C.; Barjas-Castro Mde, L.; Breitschwerdt, E.B.; et al. Bartonella clarridgeiae bacteremia detected in an asymptomatic blood donor. J. Clin. Microbiol. 2015, 53, 352-356. [CrossRef] 
17. Diniz, P.P.; Velho, P.E.; Pitassi, L.H.; Drummond, M.R.; Lania, B.G.; Barjas-Castro, M.L.; Sowy, S.; Breitschwerdt, E.B.; Scorpio, D.G. Risk factors for Bartonella species infection in blood donors from Southeast Brazil. PLoS Negl. Trop. Dis. 2016, 10, e0004509. [CrossRef] [PubMed]

18. Oteo, J.A.; Maggi, R.; Portillo, A.; Bradley, J.; García-Álvarez, L.; San-Martín, M.; Roura, X.; Breitschwerdt, E. Prevalence of Bartonella spp. by culture, PCR and serology, in veterinary personnel from Spain. Parasit. Vectors 2017, 10, 553. [CrossRef]

19. Maggi, R.G.; Mascarelli, P.E.; Pultorak, E.L.; Hegarty, B.C.; Bradley, J.M.; Mozayeni, B.R.; Breitschwerdt, E.B. Bartonella spp. bacteremia in high-risk immunocompetent patients. Diagn Microbiol. Infect. Dis. 2011, 71, 430-437. [CrossRef]

20. Maggi, R.G.; Mozayeni, B.R.; Pultorak, E.L.; Hegarty, B.C.; Bradley, J.M.; Correa, M.; Breitschwerdt, E.B. Bartonella spp. bacteremia and rheumatic symptoms in patients from Lyme disease endemic region. Emerg. Infect. Dis. 2012, 18, 783-791. [CrossRef]

21. La Scola, B.; Raoult, D. Culture of Bartonella quintana and Bartonella henselae from human samples: A 5-year experience (1993 to 1998). J. Clin. Microbiol. 1999, 37, 1899-1905. [CrossRef]

22. Bergmans, A.M.; Peeters, M.F.; Schellekens, J.F.; Vos, M.C.; Sabbe, L.J.; Ossewaarde, J.M.; Verbakel, H.; Hooft, H.J.; Schouls, L.M. Pitfalls and fallacies of cat scratch disease serology: Evaluation of Bartonella henselae-based indirect fluorescence assay and enzyme-linked immunoassay. J. Clin. Microbiol. 1997, 35, 1931-1937. [CrossRef]

23. Vermeulen, M.J.; Herremans, M.; Verbakel, H.; Bergmans, A.M.; Roord, J.J.; van Dijken, P.J.; Peeters, M.F. Serological testing for Bartonella henselae infections in The Netherlands: Clinical evaluation of immunofluorescence assay and ELISA. Clin. Microbiol. Infect. 2007, 13, 627-634. [CrossRef] [PubMed]

24. La Scola, B.; Raoult, D. Serological cross-reactions between Bartonella quintana, Bartonella henselae, and Coxiella burnetii. J. Clin. Microbiol. 1996, 34, 2270-2274. [CrossRef]

25. McGill, S.L.; Regnery, R.L.; Karem, K.L. Characterization of human immunoglobulin (Ig) isotype and IgG subclass response to Bartonella henselae infection. Infect. Immun. 1998, 66, 5915-5920. [CrossRef] [PubMed]

26. Balakrishnan, N.; Cherry, N.A.; Linder, K.E.; Pierce, E.; Sontakke, N.; Hegarty, B.C.; Bradley, J.M.; Maggi, R.G.; Breitschwerdt, E.B. Experimental infection of dogs with Bartonella henselae and Bartonella vinsonii subsp. berkhoffii. Vet. Imm. Immunopathol. 2013, 156, 153-158. [CrossRef] [PubMed]

27. Hegarty, B.C.; Bradley, J.M.; Lappin, M.R.; Balakrishnan, N.; Mascarelli, P.E.; Breitschwerdt, E.B. Analysis of seroreactivity against cell culture-derived Bartonella spp. antigens in dogs. J. Vet. Int. Med. 2014, 28, 38-41. [CrossRef] [PubMed]

28. Lashnits, E.; Correa, M.; Hegarty, B.C.; Birkenheuer, A.J.; Breitschwerdt, E.B. Bartonella seroepidemiology in dogs from North America, 2008-2014. J. Vet. Int. Med. 2018, 32, 222-231. [CrossRef] [PubMed]

29. Lashnits, E.W.; Neupane, P.; Maggi, R.G.; Linder, K.E.; Bradley, J.M.; Balakrishnan, N.; Southern, B.L.; McKeon, G.P.; Chandrashekar, R.; Breitschwerdt, E.B. Detection of Bartonella spp. in dogs after infection with Rickettsia rickettsii. J. Vet. Int. Med. 2020, 34, 145-159. [CrossRef]

30. Neupane, P.; Hegarty, B.C.; Marr, H.S.; Maggi, R.G.; Birkenheuer, A.J.; Breitschwerdt, E.B. Evaluation of cell culture-grown Bartonella antigens in immunofluorescent antibody assays for the serological diagnosis of bartonellosis in dogs. J. Vet. Int. Med. 2018, 2, 1958-1964. [CrossRef]

31. Oliveira, A.M.; Maggi, R.G.; Woods, C.W.; Breitschwerdt, E. B. Putative needle stick transmission of Bartonella vinsonii subsp. berkhoffii to a veterinarian. J. Vet. Int. Med. 2010, 24, 1229-1232. [CrossRef]

32. Edouard, S.; Nabet, C.; Lepidi, H.; Fournier, P.E.; Raoult, D. Bartonella, a common cause of endocarditis: A report on 106 cases and review. J. Clin. Microbiol. 2015, 53, 824-829. [CrossRef] [PubMed]

33. Riess, T.; Dietrich, F.; Schmidt, K.V.; Kaiser, P.O.; Schwarz, H.; Schäfer, A.; Kempf, V.A. Analysis of a novel insect cell culture medium-based growth medium for Bartonella species. Appl. Environ. Microbiol. 2008, 74, 5224-5227. [CrossRef]

34. Lynch, T.; Iverson, J.; Kosoy, M. Combining culture techniques for Bartonella: The best of both worlds. J. Clin. Microbiol. 2011, 49, 1363-1368. [CrossRef]

35. Maggi, R.G.; Duncan, A.W.; Breitschwerdt, E.B. Novel chemically modified liquid medium that will support the growth of seven Bartonella species. J. Clin. Microbiol. 2005, 43, 2651-2655. [CrossRef]

36. Duncan, A.W.; Maggi, R.G.; Breitschwerdt, E.B. A combined approach for the enhanced detection and isolation of Bartonella species in dog blood samples: Pre-enrichment culture followed by PCR and subculture onto agar plates. J. Microbiol. Methods 2007, 69, 273-281. [CrossRef] 
37. Randell, M.G.; Balakrishnan, N.; Gunn-Christie, R.; Mackin, A.; Breitschwerdt, E.B. Bartonella henselae infection in a dog with recalcitrant ineffective erythropoiesis. Vet. Clin. Pathol. 2018, 47, 45-50. [CrossRef]

38. Southern, B.L.; Neupane, P.; Ericson, M.E.; Dencklau, J.C.; Linder, K.E.; Bradley, J.M.; McKeon, G.P.; Long, C.T.; Breitschwerdt, E.B. Bartonella henselae in a dog with ear tip vasculitis. Vet. Dermatol. 2018, 29, 537-e180. [CrossRef]

39. Lantos, P.M.; Maggi, R.G.; Ferguson, B.; Varkey, J.; Park, L.P.; Breitschwerdt, E.B.; Woods, C.W. Detection of Bartonella species in the blood of veterinarians and veterinary technicians: A newly recognized occupational hazard. Vector Borne Zoonotic Dis. 2014, 14, 563-570. [CrossRef]

40. Bradley, J.M.; Mascarelli, P.E.; Trull, C.L.; Maggi, R.G.; Breitschwerdt, E.B. Bartonella henselae infections in an owner and two Papillon dogs exposed to tropical rat mites (Ornithonyssus bacoti). Vector Borne Zoonotic Dis. 2014, 14, 703-709. [CrossRef] [PubMed]

41. Rossi, M.A.; Balakrishnan, N.; Linder, K.E.; Messa, J.B.; Breitschwerdt, E.B. Concurrent Bartonella henselae infection in a dog with panniculitis and owner with ulcerated nodular skin lesions. Vet. Dermatol. 2015, 26, 60-63. [CrossRef] [PubMed]

42. Takhampunya, R.; Korkusol, A.; Pongpichit, C.; Yodin, K.; Rungrojn, A.; Chanarat, N.; Promsathaporn, S.; Monkanna, T.; Thaloengsok, S.; Tippayachai, B.; et al. Metagenomic approach to characterizing disease epidemiology in a disease-endemic environment in Northern Thailand. Front. Microbiol. 2019, 10, 319. [CrossRef] [PubMed]

43. Regier, Y.; Komma, K.; Weigel, M.; Kraiczy, P.; Laisi, A.; Pulliainen, A.T.; Hain, T.; Kempf, V.A.J. Combination of microbiome analysis and serodiagnostics to assess the risk of pathogen transmission by ticks to humans and animals in central Germany. Parasit Vectors 2019, 12, 11. [CrossRef] [PubMed]

44. El Hamzaoui, B.; Laroche, M.; Parola, P. Detection of Bartonella spp. in Cimex lectularius by MALDI-TOF MS. Comp. Immunol. Microbiol. Infect. Dis. 2019, 64, 130-137. [CrossRef]

45. Diniz, P.P.; Billeter, S.A.; Otranto, D.; De Caprariis, D.; Petanides, T.; Mylonakis, M.E.; Koutinas, A.F.; Breitschwerdt, E.B. Molecular documentation of Bartonella infection in dogs in Greece and Italy. J. Clin. Microbiol. 2009, 47, 1565-1567. [CrossRef]

46. Celebi, B.; Taylan Ozkan, A.; Kilic, S.; Akca, A.; Koenhemsi, L.; Pasa, S.; Yildiz, K.; Mamak, N.; Guzel, M. Seroprevalence of Bartonella vinsonii subsp. berkhoffii in urban and rural dogs in Turkey. J. Vet. Med. Sci. 2010, 72, 1491-1494. [CrossRef]

47. Shelnutt, L.M.; Balakrishnan, N.; DeVanna, J.; Batey, K.L.; Breitschwerdt, E.B. Death of military working dogs due to Bartonella vinsonii subspecies berkhoffii genotype III endocarditis and myocarditis. Mil. Med. 2017, 182, e1864-e1869. [CrossRef]

48. Fournier, P.E.; Gouriet, F.; Casalta, J.P.; Lepidi, H.; Chaudet, H.; Thuny, F.; Collart, F.; Habib, G.; Raoult, D. Blood culture-negative endocarditis: Improving the diagnostic yield using new diagnostic tools. Medicine 2017, 96, e8392. [CrossRef]

49. Blanco Ramos, J.R.; Oteo Revuelta, J.A.; Martínez de Artola, V.; Ramalle Gómara, E.; García Pineda, A.; Ibarra Cucalón, V. Seroepidemiology of Bartonella henselae infection in a risk group. Rev. Clin. Esp. 1998, 198, 805-809.

50. Blanco, J.R.; Oteo, J.A.; Martínez, V.; Ramalle, E.; García, A.; Ibarra, V.; Rosel, L. Seroepidemiology of Bartonella henselae infection in HIV-infected patients. Enferm. Infecc. Microbiol. Clin. 1999, 17, 434-438. [PubMed]

51. Lei, B.R.; Olival, K.J. Contrasting patterns in mammal-bacteria coevolution: Bartonella and leptospira in bats and rodents. PLoS Negl. Trop. Dis. 2014, 8, e2738. [CrossRef] [PubMed]

52. André, M.R.; Gutiérrez, R.; Ikeda, P.; do Amaral, R.B.; de Sousa, K.C.M.; Nachum-Biala, Y.; Lima, L.; Teixeira, M.M.G.; Machado, R.Z.; Harrus, S. Genetic diversity of Bartonella spp. in vampire bats from Brazil. Transbound. Emerg. Dis. 2019, 66, 2329-2341. [CrossRef] [PubMed]

53. Cheslock, M.A.; Embers, M.E. Human bartonellosis: An underappreciated public health problem? Trop. Med. Infect. Dis. 2019, 4, 69. [CrossRef]

(C) 2020 by the authors. Licensee MDPI, Basel, Switzerland. This article is an open access article distributed under the terms and conditions of the Creative Commons Attribution (CC BY) license (http://creativecommons.org/licenses/by/4.0/). 